\title{
A Posteriori Error Estimates of the Galerkin Spectral Methods for Space-Time Fractional Diffusion Equations
}

\author{
Huasheng Wang ${ }^{1}$, Yanping Chen ${ }^{1, *}$ Yunqing Huang ${ }^{2}$ \\ and Wenting Mao ${ }^{1}$ \\ ${ }^{1}$ School of Mathematical Science, South China Normal University, Guangzhou, \\ Guangdong 520631, China \\ 2 School of Mathematics and Computational Science, Xiangtan University, Xiangtan, \\ Hunan 411105, China
}

Received 13 May 2019; Accepted (in revised version) 5 October 2019

\begin{abstract}
In this paper, an initial boundary value problem of the space-time fractional diffusion equation is studied. Both temporal and spatial directions for this equation are discreted by the Galerkin spectral methods. And then based on the discretization scheme, reliable a posteriori error estimates for the spectral approximation are derived. Some numerical examples are presented to verify the validity and applicability of the derived a posteriori error estimator.
\end{abstract}

AMS subject classifications: 65M12, 65M70

Key words: Galerkin spectral methods, space-time fractional diffusion equations, a posteriori error estimates.

\section{Introduction}

Nowadays, fractional derivatives have become an important tool to describe many different types of complex mechanical and physical behaviors. Moreover, fractional calculus theory has been successfully applied in many fields such as anomalous diffusion, viscoelastic materials, geophysics and biomedical engineering. Generally speaking, fractional calculus operator is non-local, so the numerical methods which are very effective for calculating integral order differential equations may be completely invalid for fractional differential equations (FDEs). Therefore, the numerical solution of fractional differential equations has attracted more and more attention of mathematical workers $[6,12$, 20].

${ }^{*}$ Corresponding author.

Emails: huashengwang123@163.com (H. Wang), yanpingchen@scnu.edu.cn (Y. Chen), huangyq@xtu.edu.cn (Y. Huang), maowenting126@163.com (W. Mao) 
During the last decades, there appears a growing interest in developing numerical methods for solving FDEs. And the early methods mainly include finite difference method and finite element method. For example, Deng [5] discussed a finite element method for the fractional Fokker-Planck equation and a convergence rate $\mathcal{O}\left(k^{2-\alpha}+h^{\mu}\right)$ was obtained. Wang and Yang [22] studied finite element methods for variable-coefficient conservative fractional elliptic differential equations and showed that the weak formulation is well posed. Wang [21] investigated fast alternating-direction finite difference methods for three-dimensional space-fractional diffusion equations, which effectively reduced the computation and storage requirements of each iteration. Nochetto, Otárola and Salgado [19] studied the finite element approximation for parabolic equations with fractional diffusion and the stability and error estimates of the scheme were given. Jin et al. [10] analyzed Galerkin finite element methods for inhomogeneous fractional diffusion equation and the $L^{2}$ - and $H^{\frac{\alpha}{2}}$-norm error estimates were derived for the semidiscrete scheme and $L^{2}$-norm error estimates were obtained for the fully discrete schemes. Zeng et al. [28] proposed a numerical method based on a fractional linear multistep methods in time and the FEM in space for time-fractional subdiffusion equation with Dirichlet boundary conditions. Bu et al. [1] investigated finite difference/finite element method for two-dimensional space and time fractional Bloch-Torrey equations, the stability and convergence of the semidiscrete scheme and fully discrete scheme were proved. Hou, Tang and Yang [8] considered the fully discretized Crank-Nicolson scheme for fractionalin-space Allen-Cahn equations and showed that the numerical solutions satisfy discrete maximum principle under reasonable time step constraint. Recently, there are many works for the fractional differential equations [11,26]. For example, Yue et al. [27] considered a fully finite element adaptive adaptive algebraic multigrid (AMG) method for timespace Caputo-Riesz fractional diffusion equations, which have the well robustness and high efficiency compared with the classical AMG method. Xing and Wen [23] considered a class of two-dimensional Riesz space-fractional diffusion equations by the alternating direction implicit Crank-Nicholson (ADI-CN) method, which reduces the computational complexity and is unconditionally stable. Gunzburger and Wang [7] studied the time fractional partial differential equation by the Crank-Nicolson method, which achieves second-order convergence in time under the regularity assumptions of the source and initial data.

Fractional differential equations have non-local operators, which will inevitably lead to the overall dependence of numerical solutions, i.e., the full algebraic system. So the advantage of sparsity for the low-order method over the high-order method cannot be reflected. As a global high-precision algorithm, spectral methods have apparent superiority and become the preferred algorithm to solve this kind of equation. Several spectral methods for FDEs have been proposed recently, for instance Lin and $\mathrm{Xu}$ [16] proposed a numerical method based on a finite difference scheme in time and Legendre spectral method in space for time fractional diffusion equation. They also proposed a space-time spectral method for the time fractional diffusion equation and derived a priori error estimate [13]. Chen, Shen and Wang [4] considered the General Jacobi functions Petrov- 
Galerkin approximation for the fractional diffusion equation and showed that the true spectral accuracy can be attained if the data are smooth enough. Mao and Shen [18] developed Efficient Spectral-Galerkin algorithms for multi-dimensional fractional elliptic equations with variable coefficients in conserved form as well as non-conserved form and the corresponding weighted error estimation was obtained. Huang et al. [9] considered a nonpolynomial-based spectral collocation method and its well-conditioned variant are proposed and analysed. In [24], the spectral collocation methods were adopted to solve the time-fractional diffusion-wave equations and the corresponding convergence analysis was obtained. We proposed the spectral method for space-time fractional optimal control problem with state constraint and in which a priori and a posteriori error estimates were obtained [15]. As we all known, a posteriori error estimator is computable and can reflect the information of the numerical solutions. Therefore it is the key to obtain the possible optimal approximation by the least computation. There are some results of a posteriori error estimates for fractional differential problems, see reference $[2,3,25]$.

In this study, Let $\Omega:=\Lambda \times I$, where $\Lambda=(0,1)$ and $I=(0,1]$ are space and time domain respectively throughout the paper, we consider the following space-time fractional diffusion equation:

$$
\begin{cases}{ }_{0} \partial_{t}^{\alpha} u(x, t)-\lambda_{0} \partial_{x}^{\beta} u(x, t)=f(x, t), & x \in(0,1), \quad t \in(0,1] \\ u(0, t)=0, & u(1, t)=0 \\ u(x, 0)=0, & \end{cases}
$$

where the derivative operators are Riemann-Liouville derivatives and $\alpha, \beta, \lambda$ are real numbers such that $0<\alpha<1,1<\beta<2, \lambda \geq 0$.

The article layout is as follows. Some basic concepts and relevant properties of fractional derivatives are introduced in Section 2. In Section 3, the variational formulation of problem (1.1) is discussed and the existence and stability of the variational formulation are given. In Section 4, the Galerkin spectral methods are proposed to obtained the numerical solution. Moreover, a posteriori error estimates are derived. Numerical implementation and numerical examples are showed in Section 5. Finally, we conclude this article with a conclusion in Section 6.

\section{Preliminaries}

In this section, we first review some basic concepts and relevant properties of fractional derivatives and introduce the solution space, which are useful to our analysis hereafter.

Definition 2.1 (Riemann-Liouville fractional derivative, [6,20]). For $\forall x \in(a, b), s \in[n-1, n)$, $n \in \mathbb{N}$, the left-hand side and right-hand side Riemann-Liouville fractional derivative of 
order $s$ are defined respectively as follows:

$$
\begin{aligned}
& { }_{a} \partial_{x}^{s} v(x)=\frac{1}{\Gamma(n-s)} \frac{d^{n}}{d x^{n}} \int_{a}^{x} \frac{v(t)}{(x-t)^{s-n+1}} d t \\
& { }_{x} \partial_{b}^{s} v(x)=\frac{(-1)^{n}}{\Gamma(n-s)} \frac{d^{n}}{d x^{n}} \int_{x}^{b} \frac{v(t)}{(t-x)^{s-n+1}} d t
\end{aligned}
$$

where $\Gamma(\cdot)$ is the usual Gamma function.

Definition 2.2 (Caputo fractional derivative, [6, 20]). For $\forall x \in(a, b), s \in[n-1, n), n \in \mathbb{N}$, the left-hand side and right-hand side Caputo fractional derivative of order $s$ are defined respectively as follows:

$$
\begin{aligned}
& { }_{a}^{C} \partial_{x}^{s} v(x)=\frac{1}{\Gamma(n-s)} \int_{a}^{x} \frac{v^{n}(t)}{(x-t)^{s-n+1}} d t, \\
& { }_{x}^{C} \partial_{b}^{s} v(x)=\frac{(-1)^{n}}{\Gamma(n-s)} \int_{x}^{b} \frac{v^{n}(t)}{(t-x)^{s-n+1}} d t .
\end{aligned}
$$

One can verify by integration by parts that, for $\forall x \in(a, b), s \in[n-1, n)$, RiemannLiouville and Caputo fractional derivatives have the following relationships:

$$
\begin{aligned}
& \partial_{a}^{s} v(x)={ }_{a}^{C} \partial_{x}^{s} v(x)+\sum_{k=0}^{n-1} \frac{v^{k}(a)}{\Gamma(1+k-s)}(x-a)^{k-s}, \\
& { }_{x} \partial_{b}^{s} v(x)={ }_{x}^{C} \partial_{b}^{s} v(x)+\sum_{k=0}^{n-1} \frac{(-1)^{k} v^{k}(b)}{\Gamma(1+k-s)}(b-x)^{k-s} .
\end{aligned}
$$

Let $C$ be the positive real number independent of any functions. For the sake of brevity, we denote $A \lesssim B,(A \gtrsim B)$ by $A \leq C B,(A \geq C B)$ respectively. And the notation $A \cong B$ means that $A \lesssim B \lesssim A$.

Now, we recall some spaces endowed with norms. It is well known that the inner product and norm of the space $L^{2}(\Lambda)$ are defined by

$$
(u, v)_{\Lambda}=\int_{\Lambda} u v d \Lambda, \quad\|u\|_{0, \Lambda}=(u, u)_{\Lambda}^{\frac{1}{2}}
$$

respectively.

Let $C_{0}^{\infty}(\Lambda)$ be the space of smooth functions with compact support in $\Lambda$. Let $H^{s}(\Lambda)$ and $H_{0}^{s}(\Lambda)$ be the usual Sobolev spaces equipped with usual norms $\|\cdot\|_{s, \Lambda}$, where $s$ is a nonnegative real number. Then we know that the space $H_{0}^{s}(\Lambda)$ is denoted as the closure of $C_{0}^{\infty}(\Lambda)$ with respect to the norm $\|\cdot\|_{s, \Lambda}$.

Now, we define the space

$$
B^{s, \theta}(\Omega):=H_{0}^{s}\left(I, L^{2}(\Lambda)\right) \cap L^{2}\left(I, H_{0}^{\theta}(\Lambda)\right),
$$


equipped with the norm:

$$
\|v\|_{B^{s, \theta}(\Omega)}:=\left(\|v\|_{H_{0}^{s}\left(I, L^{2}(\Lambda)\right)}^{2}+\|v\|_{L^{2}\left(I, H_{0}^{\theta}(\Lambda)\right)}^{2}\right)^{\frac{1}{2}},
$$

where $\|v\|_{H_{0}^{s}\left(I, L^{2}(\Lambda)\right)}:=\|\| v(\cdot, t)\left\|_{0, \Lambda}\right\|_{s, I}\|v\|_{L^{2}\left(I, H_{0}^{\theta}(\Lambda)\right)}:=\|\| v(\cdot, t)\left\|_{\theta, \Lambda}\right\|_{0, I}$.

One can verified that $B^{s, \theta}(\Omega)$ is a Banach space.

Definition 2.3 ([14]). Let $s>0$, we define the space:

$$
H_{l}^{s}(\Lambda):=\left\{v \mid\|v\|_{H_{l}^{s}(\Lambda)}<\infty\right\},
$$

endowed with semi-norm and norm

$$
|v|_{H_{l}^{s}(\Lambda)}:=\left\|\partial_{x}^{s} v\right\|_{0, \Lambda^{\prime}} \quad\|v\|_{H_{l}^{s}(\Lambda)}:=\left(\|v\|_{0, \Lambda}^{2}+|v|_{H_{l}^{s}(\Lambda)}^{2}\right)^{\frac{1}{2}} .
$$

Definition 2.4 ([14]). Let $s>0$, we define the space:

$$
H_{r}^{s}(\Lambda):=\left\{v \mid\|v\|_{H_{r}^{s}(\Lambda)}<\infty\right\},
$$

equipped with semi-norm and norm

$$
|v|_{H_{r}^{s}(\Lambda)}:=\left\|\partial_{x} \partial_{1}^{s} v\right\|_{0, \Lambda}, \quad\|v\|_{H_{r}^{s}(\Lambda)}:=\left(\|v\|_{0, \Lambda}^{2}+|v|_{H_{r}^{s}(\Lambda)}^{2}\right)^{\frac{1}{2}} .
$$

Definition 2.5 ([14]). Let $s>0$, we define the space:

$$
H_{c}^{s}(\Lambda):=\left\{v \mid\|v\|_{H_{c}^{s}(\Lambda)}<\infty\right\},
$$

equipped with semi-norm and norm

$$
|v|_{H_{c}^{s}(\Lambda)}:=\left|\left({ }_{0} \partial_{x}^{s} v, x \partial_{1}^{s} v\right)_{\Lambda}\right|^{\frac{1}{2}}, \quad\|v\|_{H_{c}^{s}(\Lambda)}:=\left(\|v\|_{0, \Lambda}^{2}+|v|_{H_{c}^{s}(\Lambda)}^{2}\right)^{\frac{1}{2}} .
$$

The following lemma shows the relationship between the spaces $H_{l}^{s}(\Lambda), H_{r}^{s}(\Lambda), H_{c}^{s}(\Lambda)$ and $H_{0}^{s}(\Lambda)$, which can be reference in [13].

Lemma 2.1. Let $s>0, s \neq n+\frac{1}{2}$, then the semi-norms and norms of spaces $H_{l}^{s}(\Lambda), H_{r}^{s}(\Lambda), H_{c}^{s}(\Lambda)$ and $H_{0}^{s}(\Lambda)$ are equivalent in space $C_{0}^{\infty}(\Lambda)$.

For the sake of discussion of the weak formulation, the following lemmas are needed.

Lemma 2.2 ([14]). If $0<s<2, s \neq 1, w, v \in H_{0}^{\frac{s}{2}}(\Lambda)$, then

$$
\left({ }_{0} \partial_{x}^{s} w, v\right)_{\Lambda}=\left({ }_{0} \partial_{x}^{\frac{s}{2}} w, x \partial_{1}^{\frac{s}{2}} v\right)_{\Lambda} .
$$

Lemma 2.3 ([13]). If the real number $s>0$, then for $v \in H_{0}^{s}(\Lambda)$, we have

$$
\left({ }_{0} \partial_{x}^{s} v, x \partial_{1}^{s} v\right)_{\Lambda} \cong \cos (\pi s)\left\|_{0} \partial_{x}^{s} v\right\|_{0, \Lambda}^{2}
$$




\section{Variational formulation}

Now we consider the weak formulation of problem (1.1). Multiplying both sides of Eq. (1.1) by $v(x, t) \in B^{\frac{\alpha}{2}}, \frac{\beta}{2}(\Omega)$ and then integrating both sides of equation over $\Omega$, we obtain

$$
\left({ }_{0} \partial_{t}^{\alpha} u(x, t), v\right)_{\Omega}-\lambda\left({ }_{0} \partial_{x}^{\beta} u(x, t), v\right)_{\Omega}=(f, v)_{\Omega} .
$$

One can derive the following weak formulation of problem (1.1) by using Lemma 2.2: for $f \in B^{\frac{\alpha}{2}, \frac{\beta}{2}}(\Omega)^{\prime}$, find $u \in B^{\frac{\alpha}{2}, \frac{\beta}{2}}(\Omega)$ such that

$$
a(u, v)=(f, v)_{\Omega}, \quad \forall v \in B^{\frac{\alpha}{2}, \frac{\beta}{2}}(\Omega),
$$

where the bilinear form $a(u, v)$ as follows:

$$
a(u, v):=\left({ }_{0} \partial_{t}^{\frac{\alpha}{2}} u,{ }_{t} \partial_{1}^{\frac{\alpha}{2}} v\right)_{\Omega}-\lambda\left({ }_{0} \partial_{x}^{\frac{\beta}{2}} u, x \partial_{1}^{\frac{\beta}{2}} v\right)_{\Omega} .
$$

Now, we turn to proof problem (3.2) is well-posed. With the aid of the continuity and coercivity of bilinear form $a(u, v)$, we can derive the following theorem.

Theorem 3.1. For $\forall \alpha \in(0,1), \beta \in(1,2)$ and $f \in B^{\frac{\alpha}{2}}, \frac{\beta}{2}(\Omega)^{\prime}$, problem (3.2) exists a unique solution. Moreover, if $u$ is the solution of (3.2), then

$$
\|u\|_{B^{\frac{\alpha}{2}, \frac{\beta}{2}(\Omega)}} \lesssim\|f\|_{B^{\frac{\alpha}{2}, \frac{\beta}{2}(\Omega)^{\prime}}} .
$$

\section{Galerkin spectral method}

In this section, the Galerkin spectral methods are proposed to numerically solve the variational formulation (3.2). Then a posteriori error estimates for the spectral approximation is derived.

Let

$$
P_{M}^{0}(I):=P_{M}(I) \cap H_{0}^{\frac{\alpha}{2}}(I),
$$

where $P_{M}(I)$ defined by the polynomials space of degree less than or equal to $M$ with respect to time $t$. And let $P_{N}(\Lambda)$ be the polynomials space of degree less than or equal to $N$, then we denote the spectral approximation space with respect to space $x$ :

$$
P_{N}^{0}(\Lambda):=P_{N}(\Lambda) \cap H_{0}^{\frac{\beta}{2}}(\Lambda) .
$$

Let $L:=(M, N)$ and we define the space-time spectral approximation space as follows:

$$
S_{L}=P_{N}^{0}(\Lambda) \otimes P_{M}^{0}(I) .
$$


So the space-time Galerkin spectral approximation to problem (3.2) as follows: find $u_{L} \in$ $S_{L}$, such that

$$
a\left(u_{L}, v_{L}\right)=\left(f, v_{L}\right), \quad \forall v \in S_{L} .
$$

Since $S_{L}$ is a subspace of $B^{\frac{\alpha}{2}, \frac{\beta}{2}}(\Omega)$, then the well-posedness of the weak formulation (4.4) can be derived immediately.

Theorem 4.1. For $\forall \alpha \in(0,1), \beta \in(1,2)$ and $f \in B^{\frac{\alpha}{2}}, \frac{\beta}{2}(\Omega)^{\prime}$, problem (4.4) exists a unique solution. Moreover, if $u_{L}$ is the solution of (4.4), then

$$
\left\|u_{L}\right\|_{B^{\frac{\alpha}{2}}, \frac{\beta}{2}(\Omega)} \lesssim\|f\|_{B^{\frac{\alpha}{2}, \frac{\beta}{2}}(\Omega)^{\prime}} .
$$

In order to obtain the error analysis of the Galerkin spectral method, we introduce the following lemma, which can be found in [14,17].

Lemma 4.1. Let $p$, s be real numbers and such that

$$
p \neq n+\frac{1}{2}, \quad 0 \leq s \leq p .
$$

Then there exists an operator $\Pi_{p, N}^{s, 0}: H^{p} \cap H_{0}^{s} \rightarrow P_{N^{\prime}}^{s}$ such that for $\forall \varphi \in H^{\sigma} \cap H_{0}^{s}$, we have

$$
\left\|\varphi-\Pi_{p, N}^{s, 0} \varphi\right\|_{H^{v}(\Lambda)} \leq C N^{v-\sigma}\|\varphi\|_{H^{\sigma}(\Lambda)}, \quad \forall 0 \leq v \leq p \leq \sigma,
$$

where $C$ is a constant and $P_{N}^{s}$ denotes $P_{N} \cap H_{0}^{s}$.

From the above Lemma, let the operations

$$
\Pi_{\frac{\alpha}{2}, M}^{0,0}: H_{0}^{\frac{\alpha}{2}}(I) \rightarrow P_{M}^{0}(I),
$$

by $\forall v \in H_{0}^{\frac{\alpha}{2}}(I), \Pi_{\frac{\alpha}{2}, M}^{0,0} v \in P_{M}^{0}(I)$

$$
\Pi_{\frac{\beta}{2}, N}^{\frac{\beta}{2}, 0}: H_{0}^{\frac{\beta}{2}}(\Lambda) \rightarrow P_{N}^{0}(\Lambda)
$$

by $\forall v \in H_{0}^{\frac{\beta}{2}}(\Lambda), \Pi_{\frac{\beta}{2}, N}^{\frac{\beta}{2}, 0} v \in P_{N}^{0}(\Lambda)$.

Then we construct the approximation operator

$$
\Pi_{\frac{\alpha}{2}, M}^{0,0} \Pi_{\frac{\beta}{2}, N}^{\frac{\beta}{2}, 0}: B^{\frac{\alpha}{2}, \frac{\beta}{2}}(\Omega) \rightarrow S_{L},
$$

by $\forall v \in B^{\frac{\alpha}{2}, \frac{\beta}{2}}(\Omega), \Pi_{\frac{\alpha}{2}, N}^{0,0} \Pi_{\frac{\beta}{2}, M}^{\frac{\beta}{2}, 0} v \in S_{L}$.

Based on the residual error estimation, we deduce a posteriori error estimates by using the Galerkin orthogonality and Cauchy-Schwarz inequality. And a posteriori error estimates of the spectral approximation solution are given by the following theorem: 
Theorem 4.2. Let $0<\alpha<1,1<\beta<2$. $u, u_{L}$ be the solutions of problems (1.1) and (4.4), respectively. If $u \in B^{\frac{\alpha}{2}, \frac{\beta}{2}}(\Omega)$, then

$$
\left\|u-u_{L}\right\|_{0, \Omega} \leq\left\|u-u_{L}\right\|_{B \frac{\alpha}{2}, \frac{\beta}{2}(\Omega)} \lesssim \eta
$$

where

$$
\eta=\left(M^{-\frac{\alpha}{2}}+N^{-\frac{\beta}{2}}\right) \cdot\left\|\partial_{0}^{\alpha} u_{L}-\lambda_{0} \partial_{x}^{\beta} u_{L}-f\right\|_{0, \Omega}
$$

Proof. It is obvious that the left side of inequality holds. Let $e=u_{L}-u$, then $\prod_{\frac{\alpha}{2}, M}^{0,0} \Pi_{\frac{\beta}{2}, N}^{\frac{\beta}{2}, 0} e \in$ $S_{L}$. Thus we have

$$
\begin{aligned}
& \left\|u_{L}-u\right\|_{B{ }^{\frac{\alpha}{2}, \frac{\beta}{2}(\Omega)}}^{2} \lesssim a(e, e)=a\left(e, e-\Pi_{\frac{\alpha}{2}, M}^{0,0} \Pi_{\frac{\beta}{2}, N}^{\frac{\beta}{2}, 0} e\right) \\
= & a\left(u_{L}, e-\Pi_{\frac{\alpha}{2}, M}^{0,0} \Pi_{\frac{\beta}{2}, N}^{\frac{\beta}{2}, 0} e\right)-a\left(u, e-\Pi_{\frac{\alpha}{2}, M}^{0,0} \Pi_{\frac{\beta}{2}, N}^{\frac{\beta}{2}, 0} e\right) \\
= & \left({ }_{0} \partial_{t}^{\alpha} u_{L}-\lambda_{0} \partial_{x}^{\beta} u_{L}, e-\Pi_{\frac{\alpha}{2}, M}^{0,0} \Pi_{\frac{\beta}{2}, N}^{\frac{\beta}{2}, 0} e\right)_{\Omega}-\left(f, e-\Pi_{\frac{\alpha}{2}, M}^{0,0} \Pi_{\frac{\beta}{2}, N}^{\frac{\beta}{2}, 0} e\right)_{\Omega} \\
\lesssim & \left\|_{0} \partial_{t}^{\alpha} u_{L}-\lambda_{0} \partial_{x}^{\beta} u_{L}-f\right\|_{0, \Omega} \cdot\left\|e-\Pi_{\frac{\alpha}{2}, M}^{0,0} \Pi_{\frac{\beta}{2}, N}^{\frac{\beta}{2}, 0} e\right\|_{0, \Omega} .
\end{aligned}
$$

Now we turn to investigate the error $\left\|e-\Pi_{\frac{\alpha}{2}, M}^{0,0} \prod_{\frac{\beta}{2}, N}^{\frac{\beta}{2}, 0} e\right\|_{0, \Omega}$

$$
\begin{gathered}
\left\|e-\Pi_{\frac{\alpha}{2}, M}^{0,0} \Pi_{\frac{\beta}{2}, N}^{\frac{\beta}{2}, 0} e\right\|_{0, \Omega} \leq\left\|e-\Pi_{\frac{\alpha}{2}, M}^{0,0} e\right\|_{0, \Omega}+\left\|\Pi_{\frac{\alpha}{2}, M}^{0,0} e-\Pi_{\frac{\alpha}{2}, M}^{0,0} \Pi_{\frac{\beta}{2}, N}^{\frac{\beta}{2}, 0} e\right\|_{0, \Omega} \\
\lesssim\left\|e-\Pi_{\frac{\alpha}{2}, M}^{0,0} e\right\|_{0, \Omega}+\left\|e-\Pi_{\frac{\beta}{2}, N}^{\frac{\beta}{2}, 0} e\right\|_{0, \Omega} \lesssim\left(M^{-\frac{\alpha}{2}}+N^{-\frac{\beta}{2}}\right) \cdot\left\|u_{L}-u\right\|_{B \frac{\alpha}{2}, \frac{\beta}{2}(\Omega)} .
\end{gathered}
$$

And the last inequality is obtained by employing Lemma 4.1. Then the theorem is immediately obtained.

\section{Numerical results}

In this section, we present some numerical examples to show the efficiency of the proposed Galerkin spectral method. Now, we introduce the implementation of proposed method.

Let $L_{N}$ be the Legendre polynomial of degree $N .\left\{\xi_{i}\right\}_{i=0}^{N}$ be a set of Legendre-GaussLobatto nodes, which defined by

$$
\xi_{0}=-1, \quad \xi_{N}=1, \quad L_{N}^{\prime}\left(\xi_{i}\right)=0, \quad i=1, \cdots, N-1,
$$


and the associated weights of the Legendre-Gauss-Lobatto quadrature formula are denoted by $\omega_{i}, 0 \leq i \leq N$. Then the $(N+1) \times(M+1)$ Legendre-Gauss-Lobatto points $\left(x_{i}, t_{j}\right)$ in $\Omega$ and corresponding weights $\hat{\omega}_{i}^{N} \hat{\omega}_{j}^{M}, i=0, \cdots, N, j=0, \cdots, M$ are defined by

$$
\begin{aligned}
& \left(x_{i}, t_{j}\right):=\left(\frac{\xi_{i}^{N}+1}{2}, \frac{\xi_{j}^{M}+1}{2}\right), \\
& \hat{\omega}_{i}^{N}:=\frac{\omega_{i}^{N}}{2}, \quad \hat{\omega}_{j}^{M}:=\frac{\omega_{j}^{M}}{2} .
\end{aligned}
$$

Consider the problem (4.4) with numerical quadratures as follows:

$$
\left({ }_{0} \partial_{t}^{\frac{\alpha}{2}} u_{L, t} \partial_{1}^{\frac{\alpha}{2}} v_{L}\right)_{N}-\lambda\left({ }_{0} \partial_{x}^{\frac{\alpha}{2}} u_{L, x} \partial_{1}^{\frac{\alpha}{2}} v_{L}\right)_{M}=\left(f, v_{L}\right)_{L}, \quad \forall v \in S_{L}
$$

where $(\cdot, \cdot)_{N},(\cdot, \cdot)_{M}$ and $(\cdot, \cdot)_{L}$ are denoted by, for $u, v \in C(\Lambda)$

$$
\begin{aligned}
(u, v)_{N} & :=\sum_{i=0}^{N} \int_{I} u\left(x_{i}, t\right) v\left(x_{i}, t\right) \hat{\omega}_{i}^{N} d t, \\
(u, v)_{M} & :=\sum_{j=0}^{M} \int_{\Lambda} u\left(x, t_{j}\right) v\left(x, t_{j}\right) \hat{\omega}_{j}^{M} d x, \\
(u, v)_{L} & :=\sum_{i=0}^{N} \sum_{j=0}^{M} u\left(x_{i}, t_{j}\right) v\left(x_{i}, t_{j}\right) \hat{\omega}_{i}^{N} \hat{\omega}_{j}^{M} .
\end{aligned}
$$

Let $\left\{h_{i}(x)\right\}_{i=0}^{N},\left\{h_{j}(t)\right\}_{j=0}^{M}$ be the Lagrangian polynomials associated with LegendreGauss-Lobatto points $\left\{x_{i}\right\}_{i=0}^{N}$ and $\left\{t_{j}\right\}_{j=0}^{M}$ respectively. This means $h_{i}(x)$ and $h_{j}(t)$ such that

$$
h_{i}\left(x_{k}\right)=\delta_{k i}, \quad h_{j}\left(t_{k}\right)=\delta_{k j},
$$

where $\delta$ is the Kronecker function.

Then, a set of basis functions of $S_{L}$ as follows:

$$
S_{L}=\operatorname{span}\left\{h_{i}(x) h_{j}(t) ; i=1,2, \cdots, N-1 ; j=1,2, \cdots, M\right\} .
$$

Therefore, $u_{L}$ can be expressed as follows

$$
u_{L}(x, t)=\sum_{i=1}^{N-1} \sum_{j=1}^{M} u_{i j} h_{i}(x) h_{j}(t) .
$$

By taking (5.4) and letting $v_{L}$ go through all basis functions $h_{n}(x) h_{m}(t), n=1,2, \cdots, N-1$, $m=1,2, \cdots, M$ into (5.1), we can obtain the following matrix form:

$$
\mathbf{A u}=\mathbf{f},
$$


where $\mathbf{u}=\left(u_{i j}\right)_{(N-1) \times M}$ is the unknown coefficient matrix, $\mathbf{A}=\left(a_{n m, i j}\right)_{((N-1) M)^{2}}$ with

$$
\begin{aligned}
a_{n m, i j}= & \left(h_{i}(x)_{0} \partial_{t}^{\frac{\alpha}{2}} h_{j}(t), h_{n}(x)_{t} \partial_{1}^{\frac{\alpha}{2}} h_{m}(t)\right)_{N}-\lambda\left(h_{j}(t)_{0} \partial_{x}^{\frac{\beta}{2}} h_{i}(x), h_{m}(t)_{x} \partial_{1}^{\frac{\beta}{2}} h_{n}(x)\right)_{M} \\
= & \sum_{k=0}^{N} h_{i}\left(x_{k}\right) h_{n}\left(x_{k}\right) \hat{\omega}_{k}^{N} \int_{I} 0 \partial_{t}^{\frac{\alpha}{2}} h_{j}(t)_{t} \partial_{1}^{\frac{\alpha}{2}} h_{m}(t) d t \\
& \left.\quad \lambda \sum_{k=0}^{M} h_{j}\left(t_{k}\right) h_{m}\left(t_{k}\right) \hat{\omega}_{k}^{M} \int_{\Lambda} 0 \partial_{x}^{\frac{\beta}{2}} h_{i}(x)_{x} \partial_{1}^{\frac{\beta}{2}} h_{n}(x)\right) d x \\
= & \left.\delta_{i n} \hat{\omega}_{n}^{N} \int_{I} \partial_{t}^{\frac{\alpha}{2}} h_{j}(t)_{t} \partial_{1}^{\frac{\alpha}{2}} h_{m}(t) d t-\lambda \delta_{j m} \hat{\omega}_{m}^{M} \int_{\Lambda} 0 \partial_{x}^{\frac{\beta}{2}} h_{i}(x)_{x} \partial_{1}^{\frac{\beta}{2}} h_{n}(x)\right) d x,
\end{aligned}
$$

and $\mathbf{f}=\left(f_{n m}\right)_{(N-1) \times M}$ with

$$
f_{n m}=\left(f, h_{n}(x) h_{m}(t)\right)_{L}=\sum_{i=0}^{N} \sum_{j=0}^{M} h_{n}\left(x_{i}\right) h_{m}\left(t_{j}\right) \hat{\omega}_{i}^{N} \hat{\omega}_{j}^{M} .
$$

In the following, some numerical examples are given to show the a posteriori error estimates for the spectral approximation. Let $\lambda=1$ throughout the examples and the $L^{2}$-norm error $e$ and $B^{\frac{\alpha}{2}}, \frac{\beta}{2}$-norm error $E$ are defined as follows respectively,

$$
e=\left\|u-u_{L}\right\|_{0, \Omega}, \quad E=\left\|u-u_{L}\right\|_{B \frac{\alpha}{2}, \frac{\beta}{2}(\Omega)^{\prime}}
$$

and a posteriori error indicator $\eta$ is defined by

$$
\eta=\left(M^{-\frac{\alpha}{2}}+N^{-\frac{\beta}{2}}\right) \cdot\left\|\partial_{t}^{\alpha} u_{L}-\lambda_{0} \partial_{x}^{\beta} u_{L}-f\right\|_{0, \Omega} .
$$

Example 5.1. Consider the problem (1.1) with the exact solution

$$
u(x, t)=\left(x^{4}-x^{3}\right) t \sin \pi t
$$

In what follows, the errors $e, E$ and error indicator $\eta$ in $\log _{10}$ scale for varying polynomial degree $N(M=N)$ are calculated in Fig. 1. One can observe that the errors $e, E$ is exponential decay and the indicator $\eta$ has almost the same rate of convergence as the error $E$. Then the theoretical results are verified. In Fig. 2, the errors $e, E$ and a posteriori error indicator $\eta$ are showed that the numerical result still holds as $\alpha \rightarrow 1, \beta \rightarrow 2$ in this example.

Example 5.2. Consider the problem (1.1) with the exact solution

$$
u(x, t)=x \sin (\pi x) \sin ^{2}(\pi t)
$$




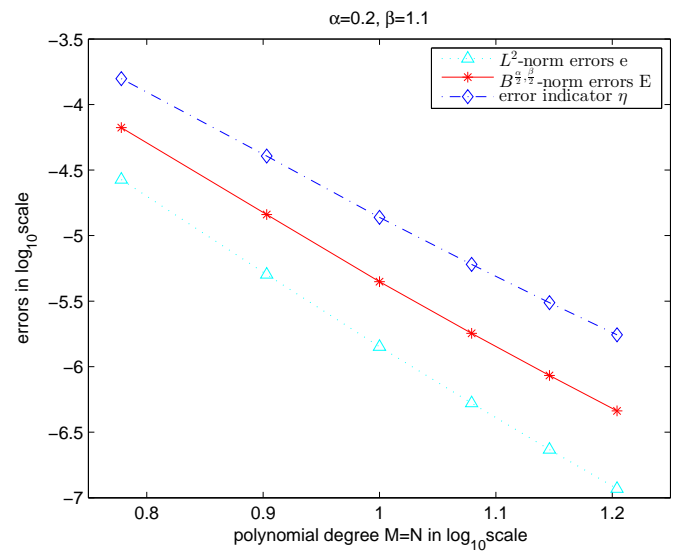

Figure 1: Error $e, E$ and error indicator $\eta$ for varying polynomial degree $N(M=N)$ with $\alpha=0.2, \beta=1.1$.

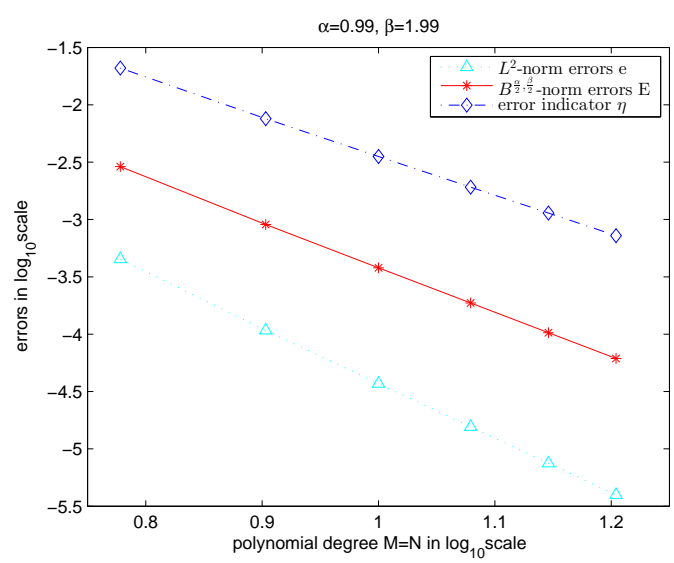

Figure 2: Error $e, E$ and error indicator $\eta$ for varying polynomial degree $N(M=N)$ with $\alpha=0.99, \beta=1.99$.

In this example, the errors $e, E$ and a posteriori error indicator $\eta$ in $\log _{10}$ scale for varying polynomial degree $N(M=N)$ with $\alpha=0.1, \beta=1.1$ are calculated in Fig. 3 . We can observe that the error $e$ and $E$ are exponential decay. Meanwhile, the curve of the indicator $\eta$ has almost coincides with the error $E$. In Fig. 4, the errors $e, E$ and a posteriori error indicator $\eta$ in $\log _{10}$ scale for varying polynomial degree $N(M=N)$ with $\alpha=0.3$, $\beta=1.1$ are also calculated, which illustrate that the obtained a posteriori error indicator is effective.

\section{Conclusions}

The Galerkin spectral methods are proposed to study the initial boundary value problem of the space-time fractional diffusion equation involving the Riemann-Liouvile fractional 


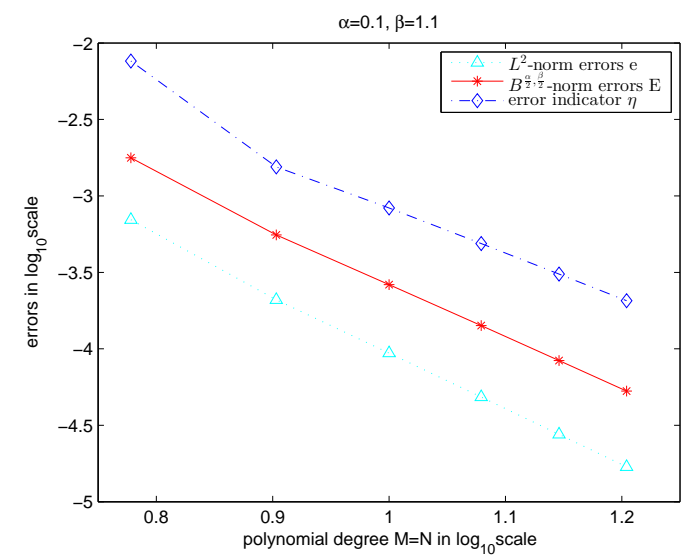

Figure 3: Error $e, E$ and error indicator $\eta$ for varying polynomial degree $N(M=N)$ with $\alpha=0.1, \beta=1.1$.

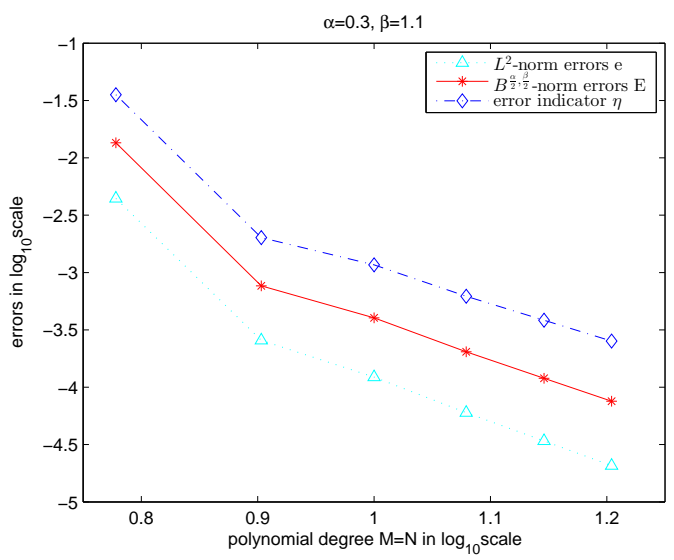

Figure 4: Error $e, E$ and error indicator $\eta$ for varying polynomial degree $N(M=N)$ with $\alpha=0.3, \beta=1.1$.

derivatives. And a posteriori error estimates for the spectral approximation are obtained. Some numerical examples are given to show the availability of the derived error indicator. In the future, an error estimator with upper and lower bounds will be considered.

\section{Acknowledgements}

This work are supported by the State Key Program of National Natural Science Foundation of China (No. 11931003) and National Natural Science Foundation of China (Nos. 41974133, 11671157 and 11971410) and supported by the Innovation Project of Graduate School of South China Normal University (No. 2018LKXM008). 


\section{References}

[1] W. BU, Y. TANG, Y. WU AND J. YANG, Finite difference/finite element method for two-dimensional space and time fractional Bloch-Torrey equations, J. Comput. Phys., 293 (2015), pp. 264-279.

[2] Z. CEN, A. LE AND A. XU, A posteriori error analysis for a fractional differential equation, Int. J. Comput. Math., 94(6) (2017), pp. 1185-1195.

[3] L. Chen, R. H. Nochetto, E. OtÁROla And A. J. SAlgado, A PDE approach to fractional diffusion: a posteriori error analysis, J. Comput. Phys., 293 (2015), pp. 339-358.

[4] S. CHEN, J. SHEN AND L. L. WANG, Generalized Jacobi functions and their applications to fractional differential equations, Math. Comput., 85(300) (2016), pp. 1603-1638.

[5] W. DENG, Finite element method for the space and time fractional Fokker-Planck equation, SIAM J. Numer. Anal., 47(1) (2008), pp. 204-226.

[6] K. Diethelm, The Analysis of Fractional Differential Equations: An Application-Oriented Exposition Using Differential Operators of Caputo Type, Springer Science \& Business Media, 2010.

[7] M. GunZburger And J. WANG, A second-order Crank-Nicolson method for time-fractional PDEs, Int. J. Numer. Anal. Model., 16(2) (2019), pp. 225-239.

[8] T. HOU, T. TANG AND J. YANG, Numerical analysis of fully discretized Crank-Nicolson scheme for fractional-in-space Allen-Cahn equations, J. Sci. Comput., 72(3) (2017), pp. 1214-1231.

[9] C. HUANG, Y. JIAO, L. L. WANG AND Z. ZHANG, Optimal fractional integration preconditioning and error analysis of fractional collocation method using nodal generalized Jacobi functions, SIAM J. Numer. Anal., 54(6) (2016), pp. 3357-3387.

[10] B. JiN, R. LAZAROV, J. PASCIAK AND Z. ZHOU, Error analysis of semidiscrete finite element methods for inhomogeneous time-fractional diffusion, IMA J. Numer. Anal., 35(2) (2014), pp. 561582.

[11] C. Li AND W. Deng, A new family of difference schemes for space fractional advection diffusion equation, Adv. Appl. Math. Mech., 9(2) (2017), pp. 282-306.

[12] C. Li AND F. ZENG, Numerical Methods for Fractional Calculus, Chapman and Hall/CRC, 2015.

[13] X. LI AND C. XU, A space-time spectral method for the time fractional diffusion equation, SIAM J. Numer. Anal., 47(3) (2009), pp. 2108-2131.

[14] X. LI AND C. XU, Existence and uniqueness of the weak solution of the space-time fractional diffusion equation and a spectral method approximation, Commun. Comput. Phys., 8(5) (2010), pp. 1016.

[15] X. LIN, Y. CHEN AND Y. HUANG, Error estimates of space-time fractional optimal control problem with state constraint, submitted, 2019.

[16] Y. LIN AND C. XU, Finite difference/spectral approximations for the time-fractional diffusion equation, J. Comput. Phys., 225(2) (2007), pp. 1533-1552.

[17] Y. MADAY, Analysis of Spectral Operators in One-Dimensional Domains, 1985.

[18] Z. MAO AND J. SHEN, Efficient spectral-Galerkin methods for fractional partial differential equations with variable coefficients, J. Comput. Phys., 307 (2016), pp. 243-261.

[19] R. H. NochetTo, E. OTÁROLA AND A. J. SALGADO, A PDE approach to space-time fractional parabolic problems, SIAM J. Numer. Anal., 54(2) (2016), pp. 848-873.

[20] I. PODLUBNY, Fractional Differential Equations: An Introduction to Fractional Derivatives, Fractional Differential Equations, to Methods of Their Solution and Some of Their Applications. Elsevier, 1998.

[21] H. WANG AND N. DU, Fast alternating-direction finite difference methods for three-dimensional 
space-fractional diffusion equations, J. Comput. Phys., 258 (2014), pp. 305-318.

[22] H. WANG AND D. YANG, Wellposedness of variable-coefficient conservative fractional elliptic differential equations, SIAM J. Numer. Anal., 51(2) (2013), pp. 1088-1107.

[23] Z. XING AND L. WEN, The fast implementation of the ADI-CN method for a class of twodimensional Riesz space-fractional diffusion equations, Adv. Appl. Math. Mech., 11(4) (2019), pp. 942-956.

[24] Y. YANG, Y. CHEN, Y. HUANG AND H. WEI, Spectral collocation method for the time-fractional diffusion-wave equation and convergence analysis, Comput. Math. Appl., 73(6) (2017), pp. 12181232.

[25] X. YE AND C. XU, A posteriori error estimates for the fractional optimal control problems, J. Inequal. Appl., 2015(1) (2015), pp. 141.

[26] S. YI AND H. SUN, A hybrided trapezoidal-difference scheme for nonlinear time-fractional fourthorder advection-dispersion equation based on chebyshev spectral collocation method, Adv. Appl. Math. Mech., 11(1) (2019), pp. 197-215.

[27] X. YUE, W. BU, S. SHU, M. LiU AND S. WANG, Fully finite element adaptive AMG method for time-space Caputo-Riesz fractional diffusion equations, Adv. Appl. Math. Mech., 10(5) (2018), pp. 1103-1125.

[28] F. ZENG, C. LI, F. LIU AND I. TURNER, Numerical algorithms for time-fractional subdiffusion equation with second-order accuracy, SIAM J. Sci. Comput., 37(1) (2015), pp. A55-A78. 